\section{Non-reciprocal X-ray linear dichroism}

\section{Gerrit van der Laan}

\author{
Daresbury Laboratory, Warrington WA4 4AD, UK. \\ E-mail: g.vanderlaan@dl.ac.uk
}

Recently, a new optical effect was observed by Goulon, Rogalev, Goulon-Ginet, Benayoun, Paolasini, Brouder, Malgrange \& Metcalf [Phys. Rev. Lett. (2000), 85, 4385-4388] using linearly polarized synchrotron radiation. The authors have correctly analysed the effect using the mathematical framework of Buckingham and Barron. A brief review is given with the underlying simplicity discussed from a symmetry point of view.

\section{Keywords: optical activity; linear dichroism; X-ray absorption.}

Optical activity was first observed in 1811 in a quartz crystal between crossed polarizers and explained as being due to optical rotation, i.e. a rotation of the plane of the linear polarization, and optical rotatory dispersion, i.e. an unequal rotation of the plane of polarization of light of different wavelengths. With intensive research continuing for almost two centuries one would not immediately expect any new discoveries to happen in this field. However, using linearly polarized $\mathrm{X}$-rays with energies of around $5 \mathrm{keV}$, Goulon et al. (2000) at the European Synchrotron Radiation Facility (ESRF) in Grenoble and his co-workers at the University of Paris and Purdue University have observed a strong non-reciprocal linear dichroism in the lowtemperature antiferromagnetic insulating phase of a Cr-doped $\mathrm{V}_{2} \mathrm{O}_{3}$ crystal in which one single antiferromagnetic domain was grown by magnetoelectric annealing. This optical effect, although theoretically predicted, has never been observed so clearly before.

Buckingham (1967) from Cambridge University, Barron (1982) from Glasgow University and others developed an elaborate mathematical framework to describe the optical activity of molecular systems in terms of spatial dispersion. Material properties can be expressed by an expansion of the optical activity tensor giving the geometrical relation between different measurable quantities. The real and imaginary part of this tensor, together with its even and odd rank, can be associated with four distinct classes of optical effects. They are listed in Table 1 and each caters for a unique experimental technique for investigating specific properties of the material. If we want to obtain a feel of the physics rather than becoming involved in the mathematics, it is sufficient to consider only the symmetry properties under space inversion and time reversal. Space inversion, represented by the parity operator $P$, inverts the coordinates of the system through the origin. It is easy to show that an electric field, $\mathbf{E}$, is represented by a polar (i.e. parity-odd) time-even vector while a magnetic field, $\mathbf{M}$, is given by an axial (i.e. parity-even) time-odd vector. As a general rule, dichroism can only exist if there is no symmetry that reverses one measurable observable but leaves the rest of the system unchanged. In the Faraday effect, noted by Michael Faraday in 1846 , the time-reversal operator, $T$, reverses both the magnetization and the direction of the light, thereby leaving the total physical system invariant. In natural circular dichroism, $P$ reverses the rotation angle (screw sense) of both the medium and the light, again leaving the total system invariant. According to Neumann's principle, these symmetries are possessed by every physical property of the material, so that non-zero effects show up only in substances that have the appropriate symmetry class. In a group-theoretical analysis of the optical activity, as has been given by e.g. Stedman (1990), this would follow from Schur's lemma. Crystal field interaction or magnetic moments of even order can induce a further symmetry breaking which is responsible for linear dichroism (LD). This requires a non-vanishing quadrupole moment, $\left\langle\mathbf{M}^{2}\right\rangle$, and has been observed by van der Laan et al. (1986) at the Tb $3 d$ absorption edges of $\mathrm{Tb}$ iron garnet.

So-called non-reciprocal optical effects invoke magnetic moments, since they are time non-invariant. They are characterized by a reversal of the phase rotation when the light propagates in the opposite direction, such as in the Faraday rotation in transmission or the Kerr effect in reflection. Also, magnetic circular dichroism (MCD), the difference in absorption for left and right circularly polarized light in the presence of an external magnetic field, belongs to this category. These effects are of great importance in studying the electronic structure, and static and dynamic processes in magnetic materials. They should be distinguished from natural optical activity that exists in the absence of an external field in an enantiomorphic system, where the atomic or molecular arrangement is not superposable onto its mirror image.

One might wonder whether these non-reciprocal optical effects are uniquely present in media possessing a net magnetic moment, such as ferro- and ferrimagnets. In all these materials the time-reversal symmetry is broken; that is, the crystal may be in two opposite magnetic states converted into each other by the time-reversal operator. Magnetoelectric antiferromagnets belong to the special class of magnetic ordered materials where there is no net magnetic moment but, in addition to time reversal, also the parity symmetry is broken, while the combined symmetry operation is conserved. The associated optical effect, non-reciprocal LD, is notoriously small in the visible region and has so far been difficult to corroborate experimentally.

Optical transitions can be expanded in multipole terms, such as electric dipole $(E 1)$, electric quadrupole ( $E 2)$, magnetic dipole $(M 1)$, together with higher-order terms that can normally be neglected. Optical activity induced by pure transitions must have even parity; only interference terms can give odd parity. Thus the $E 1 M 1$ interference in the visible region allows the detection of natural circular dichroism (CD) or optical rotation (OR) in powdered samples or in solution and single crystals. However, in the case of X-ray absorption, which involves core to valence shell excitations, the $M 1$ transitions are forbidden due to the restriction imposed by the monopole selection rule for the radial part. Although in the visible region the $E 2$ transitions are negligibly small, their magnitude increases with photon energy, so that for harder X-rays the E1E2 interference can become significant. In this way, Goulon et al. (1998) were able to observe X-ray natural circular dichroism (XNCD) at the I $2 s$ and $2 p$ edges of lithium iodate, a compound which lacks inversion symmetry. This measurement became possible thanks to the intense circularly polarized X-ray beams produced by the insertion device on beamline ID12A at the ESRF. Brouder and Natoli (Natoli et al., 1998) have shown that the measured dichroism is in agreement with theoretical model calculations.

Recently, Goulon et al. (2000) observed a non-reciprocal transverse anisotropy in the low-temperature antiferromagnetic insulating phase of a Cr-doped $\mathrm{V}_{2} \mathrm{O}_{3}$ crystal in which one single antiferromagnetic domain was grown by magnetoelectric annealing. In this phase the magnetic moments $(1.2 \mu \mathrm{B}$ per $\mathrm{V}$ atom $)$ are rotated by $71^{\circ}$ with respect to the hexagonal $c$ axis aligned along the direction of propagation of the X-rays. The space-time group $A 2$ reflects a low symmetry due to induced magnetic moments on $\mathrm{O}$ atoms: space-time 


\section{Table 1}

The optical activity tensor that describes the spatial dispersion effects in a molecular system expanded in terms of different symmetry.

The table shows their properties under space inversion $P$ and time reversal $T$, where the symbols + and - denote even and odd, respectively, which stands for invariance and sign reversal. Even and odd parity correspond to an optical tensor of even and odd rank, respectively. Even and odd reversality correspond to the real and imaginary part of the tensor, respectively. Each tensor property can be associated with a different optical effect: birefringence is the difference in the refractive index for light with opposite polarization; linear dichroism (LD) or circular dichroism (CD) is the difference in signal for light with opposite linear or circular polarization. Non-reciprocal effects lack time reversality and their observation requires another time-odd perturbation, such as an external magnetic field or exchange field. In contrast, natural circular dichroism (NCD) is a time-even phenomenon caused by a molecular dissymmetry in the absence of an external field.

\begin{tabular}{llll}
\hline$P$ & $T$ & Optical effect & Difference signal \\
\hline+ & + & Birefringence & MLD \\
+ & - & Faraday rotation & MCD \\
- & + & Optical rotation & NCD \\
- & - & Non-reciprocal birefringence & Non-reciprocal LD \\
\hline
\end{tabular}

inversion is broken and the crystal is magnetoelectric. These experiments were performed at beamline ID12A of the ESRF, where a quarter-wave plate was used to convert circularly polarized X-rays into linearly polarized X-rays with the capability to flip rapidly the polarization vector between horizontal and vertical directions. X-ray linear dichroism (XLD) spectra were recorded at the V $1 s$ edge after magnetoelectric annealing. The amplitude of the non-reciprocal XLD signal $(\sim 1 \%)$ is much stronger than that which could be measured at optical wavelengths. As expected for a time-reversal odd effect, the sign of the dichroism is reversed when the magnetic field is reversed from parallel to antiparallel with the electric field. This is the unambiguous proof that non-reciprocal XLD is inherently different from conventional X-ray magnetic linear dichroism experiments, where the magnetic anisotropy is proportional to even orders of the magnetic moment $\left\langle\mathbf{M}^{2}\right\rangle$ and, hence, remains unchanged when the direction of the magnetic field is reversed. Although the local point group $(C 3)$ at each metal centre is chiral, there is no detectable average XNCD signal because the space group I2/a belongs to a centrosymmetric crystal class. This is further evidence that the inversion symmetry is broken only as a consequence of antiferromagnetic order. Goulon et al. (2000) have correctly analysed this effect using the mathematical framework developed earlier by Buckingham (1967) and Barron (1982).

Can we now understand this effect in a simple atomic model? In the case of X-ray magnetic circular dichroism (XMCD) and X-ray magnetic linear dichroism (XMLD) the X-ray absorption process probes unoccupied valence orbitals of specific parity, e.g. in first-row transition metals the $4 p$ and $3 d$ states can be reached from the $1 s$ level with $E 1$ and $E 2$ transitions, respectively. Thole et al. (1992) derived sum rules that relate the integrated signals over the spin-orbit split absorption edges in XMCD to the spin and orbital parts of the local magnetic moment. This has led to a major breakthrough in the understanding of ferro- and ferrimagnetic materials. It is shown by van der Laan (1999) that the sum rules for XMLD provide the quadrupole moment, i.e. the charge anisotropy, and the anisotropy in the spin-orbit interaction of the valence state. However, the situation is quite different for non-reciprocal LD where the $E 1 E 2$ interference requires a substantial parity mixing between the $3 d$ and $4 p$ metal orbitals. One may hope to learn more about the orbital occupancy using the group-theoretical analysis advanced by Carra \& Benoist (2000). Already an outcome of this theory is that in the sum rule the effective operator for the orbital moment must be replaced by a dilation property as in the case of the Lorentz group. A simple atomic model is therefore at present not at hand.

Future applications include imaging of antiferromagnetic domain structures using X-ray polarization microscopy. Another possibility would be X-ray resonant scattering where the photon energy is tuned into the core to valence absorption. This gives a sensitivity to atoms at different sites in a unit cell, when the local anisotropy or gyrotropy induces additional Bragg reflections, otherwise forbidden in 'scalar' crystallography (Dmitrienko, 1983).

\section{References}

Barron, L. D. (1982). Molecular Light Scattering and Optical Activity. Cambridge University Press.

Buckingham, A. D. (1967). Adv. Chem. Phys. 12, 107-142.

Carra, P. \& Benoist, R. (2000). Phys. Rev. B, 62, R7703-R7706.

Dmitrienko, V. E. (1983). Acta Cryst. A39, 29-35.

Goulon, J., Goulon-Ginet, C., Rogalev, A., Gotte, V., Malgrange, C., Brouder, C. \& Natoli, C. R. (1998). J. Chem. Phys. 108, 6394-6403.

Goulon, J., Rogalev, A., Goulon-Ginet, C., Benayoun, G., Paolasini, L., Brouder, C., Malgrange, C. \& Metcalf, P. A. (2000). Phys. Rev. Lett. 85, $4385-4388$

Laan, G. van der (1999). Phys. Rev. Lett. 82, 640-643.

Laan, G. van der, Thole, B. T., Sawatzky, G. A., Goedkoop, J. B., Fuggle, J. C., Esteva, J. M., Karnatak, R. C., Remeika, J. P. \& Dabkowska, H. A. (1986). Phys. Rev. B, 34, 6529-6531.

Natoli, C. R., Brouder, C., Sainctavit, Ph., Goulon, J., Goulon-Ginet, C. \& Rogalev, A. (1998). Eur. Phys. J. B, 4, 1-11.

Stedman, G. E. (1990). Diagram Techniques in Group Theory. Cambridge University Press.

Thole, B. T., Carra, P., Sette, F. \& van der Laan, G. (1992). Phys. Rev. Lett. 68 , 1943-1946. 